\title{
A Novel Approach for Measurement of Total Reactive Oxidant Species (ROS) In Vivo by A Fluorometric Method
}

\author{
Boris Nemzer ${ }^{1}$, Zbigniew Pietrzkowski ${ }^{2}$, Tony Chang ${ }^{3}$, and Boxin $\mathrm{Ou}^{4}$
}

${ }^{1}$ FutureCeuticals, 300 West Sixth Street, Momence, IL 60954, USA

${ }^{2}$ Applied BioClinical Inc., 16259 Laguna Canyon Rd, Irvine, CA, 92618, USA

${ }^{3}$ International Chemistry Testing, 258 Main Street, Suite 311, Milford, MA 01757, USA

${ }^{4}$ Dover Sciences, 7 Dover Circle, Franklin, MA 02038, USA

Corresponding author:

Boxin $\mathrm{Ou}$

Dover Sciences

7 Dover Circle

Franklin, MA 02038

USA

Email: boxinou@gmail.com

Received: 5 February 2013; | Revised: 26 February 2013; | Accepted: 28 March 2013

\begin{abstract}
Six healthy participants were given a single dose of $100 \mathrm{mg}$ of CoffeeBerry® whole coffee fruit extract containing high levels of antioxidants to verify an acute effect of the treatment on ROS serum level. Blood samples were collected at $0 \mathrm{~min}, 60 \mathrm{~min}, 120 \mathrm{~min}$ and $180 \mathrm{~min}$ for subsequent measurements of serum ROS level using dihydrorhodamine 6G (DHR6G) as a fluorescent probe. The nonfluorescent DHR6G, after being oxidized by ROS present in serum samples, became rhodamine 6G (R6G) and emitted fluorescence. By quantifying R6G specific fluorescence, we were able to measure the ROS concentration. DHR 6G is indiscriminate to various free radicals (FR) found in the human body, thus DHR 6G can be very useful in quantifying total ROS in vivo. Our data indicated that five participants responded to the intake of CoffeeBerry ${ }^{\circledR}$ whole coffee fruit extract by significant decrease of ROS concentrations in vivo. Collected results are promising and indicating that DHR6G-based method could be reliable and efficacious to measure acute serum ROS changes in response to single dose treatment with antioxidant products. Therefore further clinical validation of this test is justified.
\end{abstract}

Keywords: Free radicals, CoffeeBerry®, Fluorescence Probe, DHR6G, Rhodamine 6G.

\section{Introduction}

Free radicals are consistently formed as by-products of aerobic metabolism in human body. They are generally reactive oxidant species. The most common ROS in vivo are superoxide $\left(\mathrm{O}_{2}{ }^{\circ}\right)$, hydroxyl radical $\left(\mathrm{OH}^{*}\right)$, peroxyl radical $\left(\mathrm{RO}_{2}{ }^{\circ}\right)$, nitric oxide $\left({ }^{\circ} \mathrm{NO}\right)$ and 
peroxynitrite $\left(\mathrm{ONOO}^{-}\right)[1]$. Due to their indiscriminate attacks to biomolecules such as lipids, proteins, nucleic acids and carbohydrates, they have been associated with many chronic and degenerative diseases including vascular diseases, diabetes, cancer and overall aging [2]. In order to better understand the potential mechanisms of diseases that involve ROS and to implement treatment or prevention, an in vivo analytic method to measure ROS is very important. Although there are various existing methods to assess oxidative damage caused by ROS, such as measuring lipid peroxidation products and DNA adducts, none of them evaluates ROS directly [35]. After $\mathrm{Ou}$ and Huang first demonstrated the use of DHR6G as a fluorometric assay for ROS in cigarette smoke, it logically followed that the use of the same probe to measure the ROS in biological samples should be investigated [6]. The theory behind using DHR6G is that nonfluorescent DHR6G will emit fluorescence after being oxidized by ROS. The emitted fluorescence is directly proportional to the concentration of ROS. When applied to biological samples such as plasma in the presence of the ROS, DHR6G is oxidized to highly fluorescent rhodamine $6 \mathrm{G}$. Therefore, the rhodamine $6 \mathrm{G}$ fluorescence can be used as an index to quantify the overall ROS in biological fluids. In this study, six subjects were given $100 \mathrm{mg}$ CoffeeBerry ${ }^{\circledR}$ whole coffee fruit extract. After administration, ROS in the collected serum samples were quantified by the DHR6G assay. Our results demonstrated that this fluorescent approach to measure ROS in vivo is sensitive and reliable since five subjects showed decreased ROS concentrations after intake of antioxidantrich CoffeeBerry® whole coffee fruit extract.

\section{Materials}

Chemicals: dihydrorhodamine 6G (DHR 6G) was obtained from Molecular Probes (Eugene, OR). Rhodamine 6G was purchased from Aldrich (Milwaukee, WI). HPLC grade methanol was obtained from Sigma Co. (St. Louis, MO). CoffeeBerry® whole coffee fruit extract was supplied by FutureCeuticals, Inc. (Momence, IL)
Instrument: An FL600 microplate fluorescence reader (Bio-Tek Instruments, Inc., Winooski, VT) was used with fluorescence filters for an excitation wavelength of $485 \pm 20 \mathrm{~nm}$ and an emission wavelength of $530 \pm 25 \mathrm{~nm}$. The plate reader was controlled by $\mathrm{KC} 43.0$ software.

\section{Clinical protocol}

Six healthy adult subjects were recruited into the study. The criteria for participation in the study included the following: male and female fasted, healthy subjects between the ages of 1850 , with a BMI of 25-36, free from any chronic illnesses or serious health problems, no alcohol or drug dependence, no history of organ transplantation, no surgery within the last 12 months, no medications within the last 2 weeks, no intake of supplements, and no regular consumption of coffee, green tea or other fruits (or juices). Written informed consent was obtained from each subject that participated in the trial. Peripheral venous blood samples were collected in anticoagulant-free (dry tubes) (BD Vacutainer). Before $100 \underline{180} \mathrm{mg}$ CoffeeBerry® was orally ingested, blood was collected at 'time zero" (T0). For each participant, two $9 \mathrm{~mL}$ blood samples were drawn from an antecubital vein into serum tubes. Following time zero (T0), blood was drawn at $1 \mathrm{~h}$ (T60), 2h (T120), and 3h (T180) of the treatment and always under fasting conditions. Throughout the protocol time course the subjects were advised to rest. Immediately after collection, dry tubes containing blood samples were allowed to clot and serum was collected after centrifugation at $1200 \mathrm{~g}$ for $30 \mathrm{~min}$. Serum was aliquoted, snap frozen and kept at $70^{\circ} \mathrm{C}$ until use. The study IRB \# ABC-NCI-1212-ANTX-1 was provided by Applied BioClinical, Inc. (Irvine, CA).

\section{Experimental}

Preparation of rhodamine $6 G$ standard solution: $5.15 \mathrm{mg}$ of rhodamine $6 \mathrm{G}$ was dissolved into $4 \mathrm{~mL}$ PBS buffer to make final concentration of $2.688 \mathrm{mM}$.

Preparation of DHR6G solution: $1.08 \mathrm{mg}$ of DHR6G was dissolved into $300 \mu \mathrm{L}$ DMSO to 
make a stock solution in $8.098 \mathrm{mM}$. DHR6G working solution $(202.22 \mu \mathrm{M})$ was made by adding $50 \mu \mathrm{L}$ DHR6G stock solution into 1.950 mL PBS.

Sample preparation: serum samples were thawed, vortexed, and centrifuged at 10,000rpm at $4^{\circ} \mathrm{C}$ for $3 \mathrm{~min}$. $240 \mu \mathrm{L}$ of supernatant was deproteinized using $720 \mu \mathrm{L}$ of methanol. The mixture was vortexed for 30 seconds, and then centrifuged at $10,000 \mathrm{rpm}$ for 5 minutes at $4^{\circ} \mathrm{C}$.

Fluorescence measurement: The supernatant was evaporated to dryness, and reconstituted in $60 \mu \mathrm{L}$ PBS. $40 \mu \mathrm{L}$ of above prepared sample and $60 \mu \mathrm{L}$ of DHR6G working solution were mixed well in a 96-well reading plate, and fluorescence intensity (excitation:485 nm, emission $545 \mathrm{~nm}$ ) was recorded for 24 hours with an interval of 5 minutes.

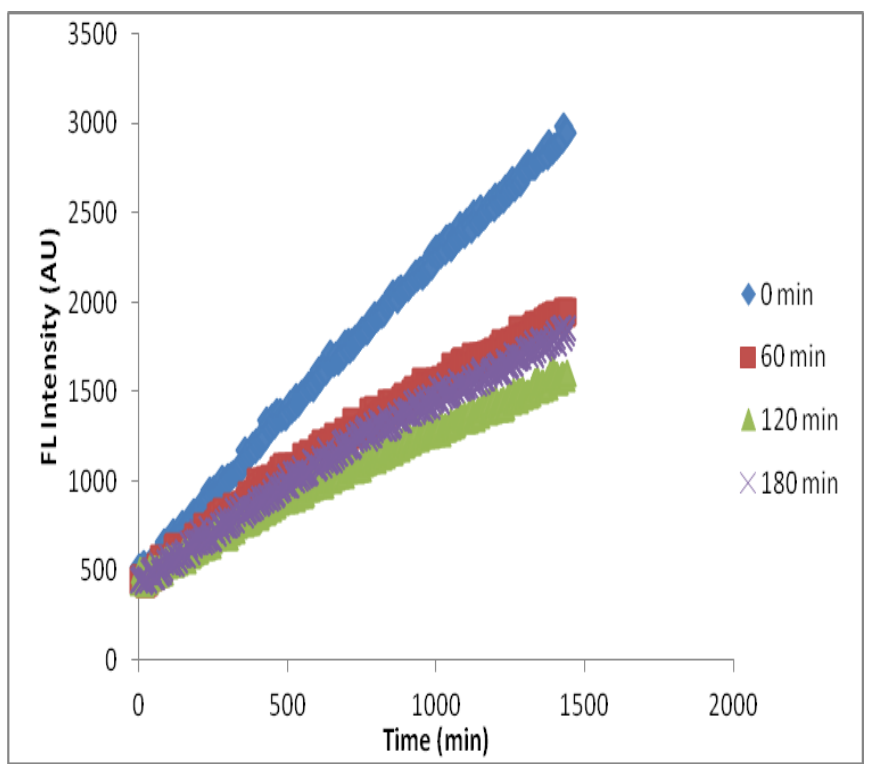

Figure 1. The changes of fluorescence intensity show the oxidation of DHR 6G was significantly inhibited after intake of $100 \mathrm{mg}$ CoffeeBerry (at $0 \mathrm{~min}$ )

\section{Results}

Oxidation of DHR6G in the presence of tested serum: DHR6G was oxidized to rhodamine $6 \mathrm{G}$ during the course of incubation with plasma extract. Figure 1 shows the fluorescence intensity increase curves over the time in serum samples collected from one subject treated with CoffeBerry®. As presented, serum collected 60 minutes post-treatment caused significant reduction (by 20-30\%) of fluorescent intensity indicating reduced level of ROS in that sample. This effect may result from presence of active components of ingested CoffeeBerry ${ }^{\circledR}$ and providing antioxidant capacity. Altogether this result indicates that CoffeeBerry ${ }^{\circledR}$ whole coffee fruit extract was bioavailable since the absorbed antioxidants neutralized endogenous free radicals. Stability of DHR6G to the air: Due to the fact that DHR-6G is labeled as "air-sensitive", we tested its stability when exposed to the air by incubating it with PBS buffer. As shown in Figure 2, during the 24-hour period, only insignificant change of fluorescence intensity was observed. On the contrary, in the presence of serum sample, the fluorescence intensity changes were apparent and linearly increased in a time sensitive manner (Figure 1).Therefore, we conclude that, under these experimental conditions, air has little contribution to any oxidation of DHR-6G; instead, the ROS in the samples should be responsible for the formation of rhodamine $6 \mathrm{G}$. From the reaction mechanism, one DHR-6G reacts with two radicals in order to form rhodamine $6 \mathrm{G}$. This finding greatly simplifies the quantitation of ROS in vivo (Figure $3)$.

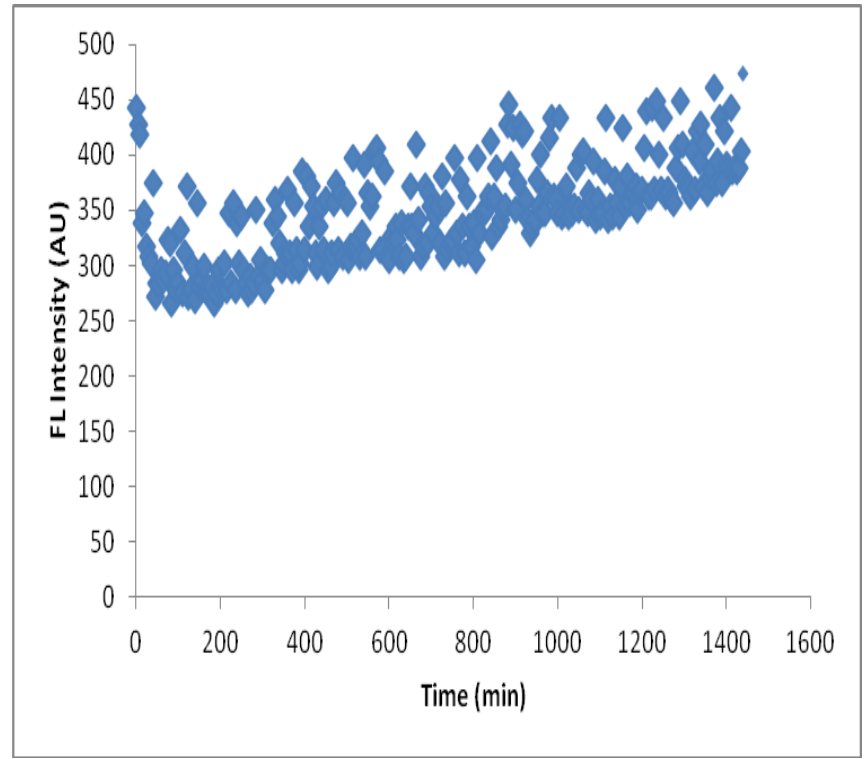

Figure 2. DHR6G at $0.406 \mu \mathrm{M}$ incubated with PBS buffer in a 96 plate well, its fluorescence intensity was monitored for 24 hours at every a 5-minute interval. Apparently, DHR6G was not auto-oxidized under the experimental condition. 
Quantitation of the free radical concentrations The concentration of rhodamine $6 \mathrm{G}$ formed in the reaction with free radicals is calculated from a standard curve obtained by plotting the known rhodamine $6 \mathrm{G}$ concentration versus the fluorescent intensity. The linearity ranges from $0.406 \mu \mathrm{M}$ to $26 \mu \mathrm{M}$ as shown in Figure 4. Using this standard curve, the concentrations of rhodamine $6 \mathrm{G}$ formed during the reaction of free radicals with DHR-6G can be calculated. The stoichiometry between DHR-6G and free radicals is two. Thus,

[Free Radicals] $=2 \times$ [rhodamine 6G] (molar concentration)

Efficacy of CoffeeBerry® in vivo. CoffeeBerry® whole coffee fruit extract's efficacy is demonstrated in Table 1 in which five subjects responded to $100 \mathrm{mg}$ CoffeeBerry® administration. The concentrations of ROS were decreased by $20 \%$ to $30 \%$ within three hours after consumption (Table 1).

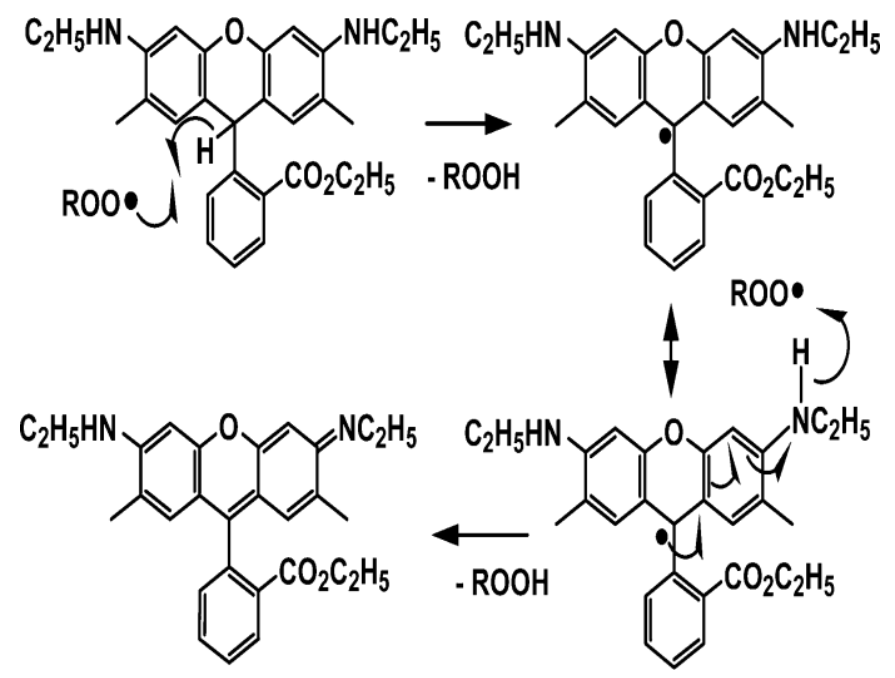

Figure 3. Mechanism of DHR6G oxidized by ROS

\section{Discussion}

By definition, free radicals are atoms or molecules, capable of independent existence, that possess one or more unpaired electrons. In the human body, there are various free radicals such as superoxide radicals, hydroperoxyl radicals, hydroxyl radicals, peroxyl radicals and alkoxyl radicals. In addition to these radicals, there are non-radical reactive species in the body, including singlet oxygen, hydrogen peroxide, peroxynitrite and hypochlorous acid. Free radicals together with non-radical reactive species are collectively terms "reactive oxidant species" (ROS). It has been suggested that excessive production of ROS can oxidize protein, damage DNA, and induce lipid peroxidation.

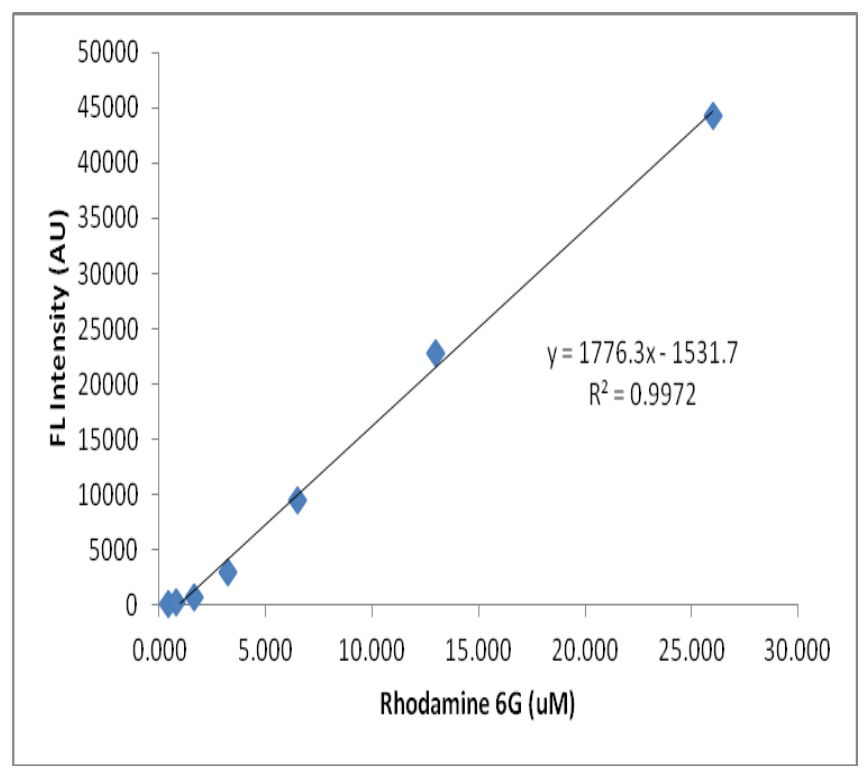

Figure 4. Standard curve of rhodamine $6 \mathrm{G}(0.406 \mu \mathrm{M}$ to $26 \mu \mathrm{M}$ )

The most frequently applied methods for measuring free radicals use spin trap and electron spin resonance. These methods are also used to detect free radicals in vivo [7]. Spin trapping coupled with ESR or HPLC dose provides direct evidence that there are free radicals in vivo, and the technique has been considered the gold standard method for free radical identification and quantitation [8]. However, the ESR-related technique is less accessible to many analytical labs due to the expensive instrument required. Therefore, there is a need to develop an alternative method to measure total oxidant species without the need for expensive instrumentation, and the method should be capable of providing a high throughput. The fluorescent probe used in the method described herein is sensitive to common ROS including 
peroxyl radicals, alkoxyl radicals, hydroxyl radicals, superoxide anion, peroxynitrite, and hypochlorous acid. Those ROS possess strong oxidizing power whereas RHD-6G is a good reductant

2ROS + DHR-6G $\longrightarrow$ rhodamine $6 \mathrm{G}+$ reduced ROS

DHR6G is inexpensive and its oxidation can be easily monitored by a fluorescence plate reader, an instrument commonly found in most analytical labs. Therefore, the protocol described in this paper could be ideal for economical quantification of the total amount of ROS in vivo. However, this method is limited in that it does not reveal individual free radicals' identity, an important factor for understanding any mechanism where free radicals are involved. For this aspect, the spin trapping technique is needed. Nonetheless, it would be ideal to combine the ESR technique with the DHR6G method for comprehensive study on identification and quantitation of free radicals.

Table 1. ROS concentrations in serum samples collected from six volunteers after intake of 100mg CoffeeBerry®

\begin{tabular}{|c|c|c|c|}
\hline Subject & Time point (min) & ROS (nM) & $\%$ Change \\
\hline \multirow{4}{*}{$\# 1$} & to & 1812 & NA \\
\hline & t60 & 1714 & -5.39 \\
\hline & $\mathrm{t} 120$ & 1752 & -3.24 \\
\hline & $\mathrm{t} 180$ & 2130 & 17.56 \\
\hline \multirow{4}{*}{$\# 2$} & to & 2500 & NA \\
\hline & t60 & 2130 & -14.83 \\
\hline & $\mathrm{t} 120$ & 1854 & -25.84 \\
\hline & $\mathrm{t} 180$ & 1538 & -38.48 \\
\hline \multirow{4}{*}{$\# 3$} & to & 2328 & NA \\
\hline & t60 & 1844 & -20.78 \\
\hline & $\mathrm{t} 120$ & 1862 & -19.99 \\
\hline & $\mathrm{t} 180$ & 2372 & 1.95 \\
\hline \multirow{4}{*}{ \#4 } & to & 2202 & NA \\
\hline & $\mathrm{t} 60$ & 2434 & 10.53 \\
\hline & $\mathrm{t} 120$ & 1848 & -16.02 \\
\hline & $\mathrm{t} 180$ & 1747 & -20.67 \\
\hline \multirow{4}{*}{$\# 5$} & to & 2084 & NA \\
\hline & t60 & 2070 & -0.68 \\
\hline & $\mathrm{t} 120$ & 1840 & -11.65 \\
\hline & $\mathrm{t} 180$ & 1566 & -24.82 \\
\hline \multirow{4}{*}{$\# 6$} & t0 & 2384 & NA \\
\hline & t60 & 1816 & -23.82 \\
\hline & $\mathrm{t} 120$ & 1894 & -20.55 \\
\hline & $\mathrm{t} 180$ & 1740 & -27.01 \\
\hline
\end{tabular}


CoffeeBerry ${ }^{\circledR}$ whole coffee fruit extract is commercially available, is rich in chlorogenic acids, and possesses high antioxidant capacity as measured by the total ORAC [9]. Higher longterm coffee consumption has consistently been associated epidemiologically with a reduced incidence of type 2 diabetes [10]. Epidemiology also suggests that coffee consumption might inhibit inflammation and thereby reduce the risk of cardiovascular and other inflammatory diseases in postmenopausal women [11]. Although antioxidant capacity of coffee polyphenols has been proposed as one of several potential in vivo mechanisms for disease prevention, evidence in human studies from this aspect is limited. This is due to the lack of appropriate analytical methods. The current methodology on antioxidant in vivo efficacy largely relies on LC/MS specific for antioxidant metabolites; however, those metabolites are usually at ultralow concentrations that make quantitation difficult. Additionally, they do not reflect the potential health benefits of intake of antioxidants. Logically, in vivo antioxidant efficacy can be determined from two dimensions: total antioxidant capacity and general ROS concentrations. Nevertheless, development of a meaningful analytical method aimed at these two aspects has the potential to stimulate better research and product formulation. To date, we are unaware of any human studies reporting any efficacy of coffee fruit extract in terms of impact on in vivo ROS concentrations. Accordingly, we report here the use of DHR6G as a novel approach using CoffeeBerry ${ }^{\circledR}$ as an example. Our results showed that the plant based antioxidants present in CoffeeBerry® whole coffee fruit extract functioned well in vivo, and that the DHR6G assay can be a potentially valuable new tool for in vivo oxidative stress studies and simple way to measure ROS in small volume of serum $(500 \mu \mathrm{l})$. We are in the process of applying the DHR 6G to another, larger scale clinical study in order to further validate this new assay. Meanwhile, possible correlations between total antioxidant capacity and total ROS concentrations are under investigation.

\section{References}

1. Halliwell, B., Reactive species and antioxidants. Redox biology is a fundamental theme of aerobic life. Plant Physiol 2006, 141, 312-22. DOI: 10.1104/pp.106.077073

2. Finkel, T.; Holbrook, N. J., Oxidants, oxidative stress and the biology of ageing. Nature 2000, 408, 239-47. DOI: 10.1038/35041687

3. Therond, P.; Bonnefont-Rousselot, D.; DavitSpraul, A.; Conti, M.; Legrand, A., Biomarkers of oxidative stress: an analytical approach. Curr Opin Clin Nutr Metab Care 2000, 3, 373-84. DOI: 10.1097/00075197-200009000-00009

4. Prior RL, Hoang H, Gu L, Wu X, Bacchiocca M, Howard L, et al., Assays for hydrophilic and lipophilic antioxidant capacity (oxygen radical absorbance capacity (ORAC(FL))) of plasma and other biological and food samples, J Agric Food Chem, 2003, 51(11),3273-79. DOI: 10.1021/jf0262256

5. Blacker BC, Snyder SM, Eggett DL, Parker TL, Consumption of blueberries with a highcarbohydrate, low-fat breakfast decreases postprandial serum markers of oxidation, $\mathrm{Br} J$ Nutr, 2012,1-8. DOI: $\underline{10.1017 / S 0007114512003650}$

6. Ou, B, Huang, D, Fluorescent Approach to Quantitation of Reactive Oxygen Species in Mainstream Cigarette Smoke, Anal. Chem. 2006; 78, 3097-3103. DOI: 10.1021/ac051993s

7. Quaresima, V, Ferrari, M, Current status of electron spin resonance (ESR) for in vivo detection of free radicals, Phys. Med. Biol. 43, 1937-1939.

8. Villamena, FA, Zweier, JL. Detection of Reactive Oxygen and Nitrogen Species by EPR Spin Trapping, Antioxidants \& Redox Signaling 2004, 6(3): 619-629. DOI: $\underline{10.1089 / 152308604773934387}$

9. Mullen, W, Nemzer, B, Ou, B, Stalmach, A, Hunter, J, Clifford, MN, Combet, E. The antioxidant and chlorogenic acid profiles of whole coffee fruits are influenced by the extraction procedures, J Agric Food Chem.; 2011, 59, 37543762. DOI: 10.1080/10408390500400009

10. Higdon, J. V, Frei, B Coffee and health: a review of recent human research, Crit. Rev. Food Sci. Nut. 2006, 46, 101-123. 
11. Andersen, LF, Jacobs, DR., Carlsen, MH, Blomhoff, $\mathrm{R}$ Consumption of coffee is associated with reduced risk of death attributed to inflammatory and cardiovascular diseases in the Iowa Women's Health Study, Am. J. Clin. Nutr. 2006, 83, 1039-1046. 